\title{
Lifetime Prediction for A Certain Missile Based on Field Data
}

\author{
Xuecheng Zhang ${ }^{*}$, Xuning Sun and Qixun Liu \\ Contract Management Office in Shanghai; Shanghai, 201109, China \\ xc-zhang@outlook.com
}

\begin{abstract}
A statistical procedure of the lifetime prediction for a certain missile is established by the reliability analysis using the field data. First, the reliability at given time point is estimated by the Kaplan-Meier method according to samples undergoing long time storage. Second, the lifetime distribution mode is obtained by fitting the relationship of the reliability and time with linear regression method. Third, parameters of the lifetime distribution are calculated by the Newton-Raphson method. Finally, the lifetime of a certain missile is predicted with the right censored field data. Results show that the field-reliability method could provide a clear reference model to estimate the lifetime of a certain missile through the field data and give some useful feedbacks for future design improvements.
\end{abstract}

Keywords: Lifetime prediction; Reliability analysis; Nonparametric statistics; Field data.

\section{Introduction}

Generally, missiles are in the storage condition until launch/destroy after they leave the factory. The complexity increases while the service life becomes much longer, for example, it is required for some newer missile systems to perform a good function even after 10 years of storage. Meanwhile, periodic maintenance and checkouts are being reduced, even to maintenance free. Reliability is one of the most important characteristics for the long-storage and one-shot missile system. Therefore the reliability estimation is important to ensure that missiles could withstand long periods of storage with high level of readiness. In such a context, the designers also want to gain some helpful feedbacks for a better design from the reliability of the similar products.

Lots of attempts had been carried out to predict storage reliability of missiles. In 1970's, the U.S. AMRDC conducted a program for reliability prediction of missile materials [1]. The U.S. navy explored the storage test data collected over 15 years of Harpoon anti-ship missile [2]. The U.S. AAMRDEC developed a health monitoring system to monitor and assess reliability of the missiles during long-term storage and field exposures [3]. Those estimation methods are often based on large amount of the lifetime data obtained through life test or field data. The accelerated stress test is commonly used to predict the reliability of a long life product. This method is economic and time-saving. However, the test could not simulate all the various working conditions. Furthermore, it has been more and more difficult to obtain the lifetime data. For the life testing, fewer even zero failures happen frequently because the system's reliability gets higher with the development of science and technology. On the other hand, the field data could reveal the actual and practical reliability of a product. The periodic check is performed to monitor the status of a certain missile. And the missile is usually tested before launching. Consequently a large number of field data are gathered for a certain missile, which equal to the life test information under the full conditions with the total number products. The reliability analysis through field data could show the status of a product and also reveal the failure rate in the future years. Therefore it is much more reasonable to estimate the storage reliability by using field data coordinating with statistical method.

The paper focuses on the lifetime prediction of a certain missile system by the reliability analysis through the field data. The rest of the paper is arranged as follows: Section II introduces the filed-reliability estimation process of the lifetime prediction by means of the field data, Section III illustrates results for the method applied to a certain missile under right censored conditions and Section IV is the conclusion. 


\section{Field-reliability Lifetime Estimation Process}

Reliability Estimation. The field data originate from periodic checking in storage, testing before/during launching. They may accord with the actual working conditions to a higher degree. But the collected data have some differences from the special samples for the parametric analysis of reliability estimation. Apparently, they couldn't reflect some important information, such as failure numbers of the same batch product, storage time, the actual failure time and the effect of storehouse environment on the product. It is feasible to derive the distribution functions by the exploration of the data using nonparametric statistics method, which does not rely on the restrictive assumption of a certain type of parametric lifetime distribution. The estimation accuracy of nonparametric method is not very high. However, it is enough for engineering application in such conditions that failure numbers are determined but the actual failure time is missing. Hence the Product Limit Method, also called Kaplan-Meier Estimator, is chosen to evaluate the reliability value.

Let assume $t_{i}$ denote the $i$ th $(i=1,2, \cdots, n)$ observation time from total $n$ independently and identically distributed samples complying with the sequence $t_{1} \leq t_{2} \leq \Lambda \leq t_{n}, d_{j}$ be the number of failures at time $t_{j}$, and $n_{j}$ be the number of items working properly just before $t_{j}$. According to Kaplan and Meier [4], the estimate of the reliability $R(t)$ can be defined as

$$
\hat{R}(t)=\prod_{\left\{j \mid t_{j} \leq t\right\}}\left[\frac{n_{j}-d_{j}}{n_{j}}\right]
$$

And the variance estimation is given as follows

$$
\operatorname{Var}[\hat{S}(t)]=\hat{S}(t)^{2} \sum_{\left\{j t_{j} \leq t\right\}} \frac{d_{j}}{n_{j}\left(n_{j}-d_{j}\right)}
$$

The reliability or survival probability at time interval could be calculated through the above two equations. And they also could be used to predict the probability of the failure at the future given time. Since the reliability means a probability, it is necessary to evaluate the tolerance limits of the estimated probability value. Reliability practitioners have proved the tolerance and confidence limits by the mathematical methods [5]. For a two-sided limit on reliability, the interval is

$$
\begin{aligned}
R_{L} & =\frac{1}{1+\frac{r+1}{n-r} F_{1-\alpha / 2}(2(r+1), 2(n-r))} \\
R_{U} & =\frac{1}{1+\frac{r}{n-r+1} F_{\alpha / 2}(2 r, 2(n-r+1))}
\end{aligned}
$$

Where $R_{L}$ is the lower limit on the reliability and $R_{U}$ is the upper limit, $r$ is the number of failures, $F_{\alpha}(u, v)$ denotes the $\alpha$ quantile of an $F$-distribution with $u$ and $v$ degrees of freedom. The probability depends on $r, n$ and $\alpha$. Similarly, a one-side limit on the reliability could be obtained by replacing the $R_{L}$ 's significance level $\alpha / 2$ with $\alpha$ in Eq.3.

Lifetime Distribution Determination. Investigations show that most failures of a certain missile system comply with the bathtub curve. In general, the infant morality has been removed to reduce the possibility of failure early in its life. Hence it is reasonable to focus on its "useful life" stage. In view of engineering application, the failure is a function of time. Furthermore, the common continuous failure distribution can be linearized. As a result, the relationship of reliability and time could be deduced by linear regression using the least square method.

$$
\hat{R}_{i}=\hat{a}+\hat{b} t_{i}
$$

The linear regression for reliability and time could be done by using Eq.4 with the common distribution patterns, such as the Weibull, exponential, log-normal and normal distribution. The goodness of fitting is determined by the correlation coefficient because the distribution is heavily skew toward the higher coefficient. The optimum failure distribution is thus found. And then parameters of the lifetime distribution should be estimated. 
Parameters Estimation. Let $f(t)$ be the probability density function (pdf) of the lifetime distribution. The likelihood function with right-censored failure time is given as follows

$$
L(t)=\prod_{i=1}^{n}[f(t)]^{v_{i}}[R(t)]^{1-v_{i}}
$$

Where $v_{i}$ is an indicator variable for each observation time $t_{i}, v_{i}=1$ denotes the failure time and $v_{i}=0$ is censored data.

To simply calculation, we rewritten the above equation into the logarithmic form as

$$
l=\sum_{i=1}^{n}\left[v_{i} \ln \left(f\left(t_{i}\right)\right)+\left(1-v_{i}\right) \ln \left(R\left(t_{i}\right)\right)\right]
$$

The likelihood equation set is sequentially obtained by differentiating Eq. 6 with respect to the unknown distribution parameters and putting them equal to zero. The estimates of distribution parameters could be solved by the Newton-Raphson method with mathematical software such as Matlab.

Lifetime Prediction. Since parameters have been determined, the average life or Mean Time To Failure (MTTF) could be calculated according to characteristics of the lifetime distribution. The lifetime $\mathrm{T}$ at given confidence level CL and lower limit on the reliability also could be predicted by the following equation

$$
P\left\{R(T) \geq R_{L}\right\}=C_{L}
$$

\section{Results and Discussion}

The results of the above method applied to a certain missile system are presented and discussed in this section. The data set are collected from tests in storage and before launch. Collecting field test data of 845 and obtain about 60 failure data. We reorganize the censored failure data into intervals divided by half of a year and reorder them from the smallest time to the largest one. The reliability at the divided time period is calculated by Eq.1. And then linear regression for the reliability and time is done by the least square method. The Weibull distribution, which is expressed as $W(\eta, \beta)$ with the scale parameter $\eta$ and the shape parameter $\beta$, fits observed data best because it has the highest correlation coefficient. It accords with the study result that the Weibull distribution is efficient to determine the life of a integrated product with both electronic and mechanical parts [6].

The computed results indicate that the confidence level and sample size influence the reliability significantly, shown in Fig. 1 and Fig. 2 respectively. This result accords with engineering experiences and also indicates that the method is feasible for the reliability estimation of a certain missile system.

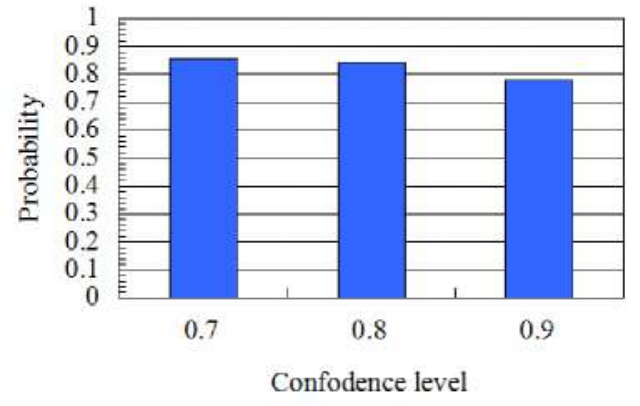

Figure 1. Effect of confidence level

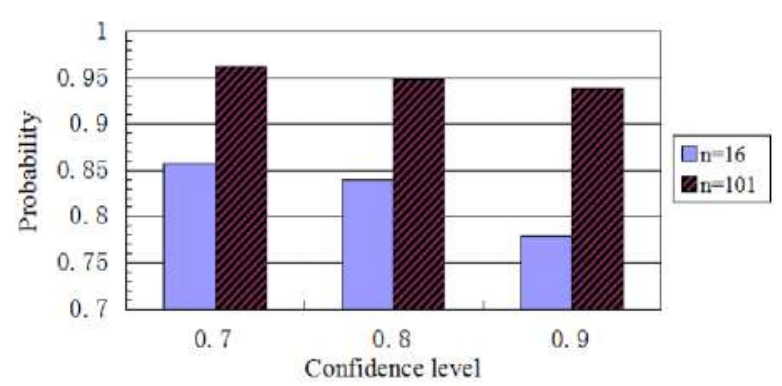

Figure 2. Effect of sample size

And there are some notices when the method is used to estimate the reliability from field data. They include:

a) The estimated value of lower limit on the reliability decreases with the increasing of confidence level for the same data set, shown in Fig.1. Therefore, it is necessary to adjust the confidence level with the sample size dynamically, for example, a low level 0.7 is chosen for the small sample size while 0.9 is chosen for the big sample size. 
b) The identical sample size should be selected for the different batches at the same place. Fig. 2 shows that the estimation precision increases with the sample size. But the inequable size may result in conservative estimate. Let fourteen samples be selected from batch A and six from batch B, and zero failure happen, the estimation results are 0.9175 and 0.8181 respectively at confidence level 0.7 . Apparently it is contrary with the engineering experience because excessive small size from batch B causes the conservative estimate.

The estimates of Weibull distribution' s parameters are $\eta=12.58$ and $\beta=1.452$. Consequently, the average life of a certain missile according to the given test data is drawn from MTTF $=\eta * \Gamma\left(1+\frac{1}{\beta}\right)=11.4$ a. The result tallies with the actual situation. Table 1 shows the corresponding lifetime estimation $T$ at given reliability value $R$, which reveals the time regularity of the storage reliability that the reliability deduces with the increasing of storage time.

Table 1. The field-reliability lifetime estimation

\begin{tabular}{|c|c|c|c|c|c|c|}
\hline Para. & \multicolumn{6}{|c|}{ Value } \\
\hline$R$ & 0.70 & 0.75 & 0.80 & 0.85 & 0.90 & 0.95 \\
\hline$T$ & 6.2 & 5.3 & 4.5 & 3.6 & 2.7 & 1.6 \\
\hline
\end{tabular}

\section{Summary}

The proposed estimation procedure deduces the mold of lifetime distribution from field data by a nonparametric method and sequentially solves the estimation of parameters as well as characteristic values of reliability. Results applied to a certain missile show that this field-reliability method could provide a clear reference model to estimate the lifetime through field data. It puts particular emphasis on the engineering practice to excavate and utilize field data. It solves the shortage of life data in reliability estimation and prediction. And the gained knowledge is useful for improving product design. A system may compose of several subsystems or components while this proposed method only utilizes the entire system' s performance. In future works, Bayesian inference could be used to integrate the component and system tests data for much better estimation of the reliability and lifetime.

\section{References}

[1] Dennis F. Malik, Storage Reliability of Missile Materiel Program. Storage Reliability Analysis Summary Report. Volume 1. Electrical and Electronic Devices, Defense Technical Information Center, 1976, pp.18-23.

[2] D.J. Theunissen, Holbrook and R. Owen, "Effects of environment and aging upon missile reliability," in Reliability and Maintainability Symposium, Proceedings., Annual. Anaheim, CA 1998, pp.314 - 320 .

[3] S.A. Marotta, A.J. Kudiya, T.K. Ooi, J.A. Gilbert and H.A. Toutanji, "Predicting reliability of tactical missiles using health monitoring data and probabilistic engineering analyses," in: SEM Annual Conference \& Exposition on Experimental and Applied Mechanics, Sep.2005, pp.1-9.

[4] R. C. Millar, T. A. Mazzuchi and S. Sarkani, "Nonparametric statistical analysis of the reliability of a naval aviation propulsion system," Naval Engineers Journal, Vol.2, Dec.2009, pp. 111-123.

[5] V. Zardasht, P. Zeephongsekul and M. Asadi, "On nonparametric estimation of a reliability function," Communications in Statistics - Theory and Methods, Vol.41, 2012, pp.983-999.

[6] Debasis Kundu, "On hybrid censored Weibull distribution," Journal of Statistical Planning and Inference, vol. 137, no. 7, pp. 2127-2142, 2007. 